\title{
Diet-induced obesity reduces the production of influenza vaccine-induced antibodies via impaired macrophage function
}

\author{
W.-J. CHO ${ }^{1}$, D.-K. LEE ${ }^{1}$, S.-Y. LEE', S.-H. SOHN ${ }^{1}$, H.-L. PARK', Y.-W. PARK², H. KIM², J.-H. NAM ${ }^{*}$
}

\begin{abstract}
${ }^{1}$ Department of Biotechnology, The Catholic University of Korea, 43-1 Yeokgok 2-dong, Wonmi-gu, Bucheon, Gyeonggi-do, 420-743, Korea; ${ }^{2}$ SK Chemical, Sampyeong-dong, Bundang-gu, Seongnam-si, Gyeonggi-do, 463-400, Korea
\end{abstract}

Received July 24, 2015; revised November 5, 2015; accepted August 8, 2016

\begin{abstract}
Summary. - Obesity is a metabolic disease characterized by low-level chronic inflammation. Obese individuals are susceptible to infection by viruses, and vaccination against these pathogens is less effective than in nonobese individuals. Here, we sought to explore the immunological environment in a mouse model of obesity induced by a high-fat diet (HFD). HFD treatment increased the body weight and epididymal fat mass. The proportion of activated B cells, T cells, and macrophages was similar between mice in the HFD group and the regular-fat diet (RFD) group. The Th1 cell subpopulation in the HFD group was increased, whereas the proportion of Treg cells was reduced compared with the RFD group. Moreover, T-cell proliferation and cytokine production did not differ between the groups when cells were stimulated with anti-CD3 and anti-CD28 antibodies in vitro. In macrophages, phagocytic activity was higher in mice fed an HFD than in those fed an RFD, but expression levels of CD86 and MHC class II antigens were similar. When macrophages were cultured in vitro, the proportion of CD86-expressing macrophages was lower in those isolated from mice in the HFD group than in those isolated from the RFD group. Furthermore, lipopolysaccharide-induced interleukin 6 (IL-6) and tumor necrosis factor alpha secretions were significantly reduced in macrophages isolated from the HFD group. In addition, influenza vaccine-induced antibodies in the HFD group diminished more rapidly than in the RFD group. These results suggest that poor functionality of macrophages during obesity might contribute to a reduction in vaccine efficacy.
\end{abstract}

Keywords: high-fat diet; macrophage; obesity; vaccine efficacy

\section{Introduction}

Obesity is the accumulation of excessive body fat caused by hypertrophy and hyperplasia of adipocytes (Jo et al., 2009). Adipose tissue is complex, composed of various cell types including adipocytes and immune cells, and it secretes

*Corresponding author. E-mail: jhnam@catholic.ac.kr; phone: +82-2-2164-4917.

Abbreviations: $\mathrm{CBV}=$ cell-culture-based vaccine; $\mathrm{CFSE}=\mathrm{Car}-$ boxyfluorescein succinimidyl ester; DIO = diet-induced obesity; FITC $=$ fluorescein isothiocyanate; HFD $=$ high-fat diet; IL = interleukin; $\mathrm{MHC}=$ major histocompatibility; LPS = lipopolysaccharide; $\mathrm{NP}=$ nucleoprotein; $\mathrm{PBSA}=\mathrm{PBS}$ bovine serum albumin; $\mathrm{PBS}=$ phosphate-buffered saline; RFD = regular-fat diet; TNF- $\alpha=$ tumor necrosis factor alpha adipokines to regulate nutrient homeostasis (Rosen and Spiegelman, 2006; Kanneganti and Dixit, 2012). In an obese environment, the population of immune cells in adipose tissue is increased, and more of them produce pro-inflammatory cytokines such as tumor necrosis factor alpha (TNF- $\alpha$ ) and interleukin (IL) 6 than in the normal state, thereby inducing low-grade chronic inflammation (Hotamisligil et al., 1995; Weisberg et al., 2003). This inflammation is considered to be a cause of leptin and insulin resistance. Leptin is a multifunction cytokine that regulates immune cell behavior such as survival, proliferation, and differentiation (Loffreda et al., 1998; Lord et al., 1998; Mattioli et al., 2005).

In mouse models of obesity such as those induced by diet, the leptin-deficiency genotype $(o b / o b)$, and leptin receptordeficiency genotype $(d b / d b)$ are more susceptible to bacterial and viral infections (Webb et al., 1976; Ikejima et al., 2005; 
Smith et al., 2007). More obese people were hospitalized and died during the $2009 \mathrm{H} 1 \mathrm{~N} 1$ influenza pandemic than did lean people (Cho and Nam, 2014). Moreover, vaccineinduced antibody production is reduced in obese mice compared with lean mice (Kim et al., 2009). Many studies have been conducted to determine the effect of obesity on immune responses. In obese mice, macrophage infiltration into adipose tissue is increased, and infiltrated macrophages are polarized to an M1 phenotype that produces pro-inflammatory cytokines causing systemic inflammation. In addition, macrophages isolated from obese rats showed lower phagocytic activity compared with those of normal rats (Plotkin and Paulson, 1996). The capacity for antigen presentation and allogeneic T-cell stimulation is reduced in dendritic cells from obese mice (Macia et al., 2006). However, these findings might not reflect the immune response capacity of obese humans because these genetic animal models of obesity showed disrupted leptin signaling.

Here we sought to analyze the overall functionality of immune cells in a diet-induced model of obesity. The activated states of macrophages, $\mathrm{B}$ and $\mathrm{T}$ cells, and the reactivity of $\mathrm{T}$ cells were similar in both normal and obese mice, whereas cytokine production by macrophages stimulated with lipopolysaccharide (LPS) was reduced in mice consuming a high-fat diet (HFD) compared with those consuming a regular-fat diet (RFD). Thus, it is possible that the reduced macrophage function might contribute to a reduction in vaccine efficacy.

\section{Materials and Methods}

Mice Four-week-old male C57BL/6 mice (Daehan Bio Link, Korea) were fed a $60 \%$ or $5 \%$ fat diet (Dooyeol Biotech, Korea) for 10 weeks. Mice ( $n=3-5 /$ group) were housed under specificpathogen-free conditions and maintained according to protocols approved by the Institutional Animal Care and Use Committee, Sungsim Campus, Catholic University of Korea. Vaccination was conducted using $9 \mu \mathrm{g}$ of cell-culture-based vaccine (CBV, SKYCellflu, SK Chemicals; Korea) by intramuscular injection after 10 weeks of the HFD. Body weights and food intake were checked, and serum samples were collected from a facial vein weekly.

Isolation and stimulation of peritoneal macrophages Peritoneal fluid was obtained by injecting cold sterile phosphate-buffered saline (PBS) into the peritoneal cavity using a $10 \mathrm{ml}$ syringe, and then gently massaged to loosen cells. Peritoneal fluid was extracted on the opposite side of the mouse using an $18 \mathrm{G}$ needle, and the procedure was repeated three times. Peritoneal fluid was centrifuged at $800 \times \mathrm{g}$ for $5 \mathrm{~min}$ at $4^{\circ} \mathrm{C}$, and the supernatant was discarded. The cell pellet was washed using PBS, incubated with red blood cell lysis buffer (Becton Dickinson (BD) Bioscience, USA) for $2 \mathrm{~min}$, centrifuged at $1500 \mathrm{rpm}$ for $5 \mathrm{~min}$ at $4^{\circ} \mathrm{C}$, and washed twice with PBS. To determine the state of macrophage activation, cells were stained with fluorescent-labeled antibodies against F4/80, CD86, and MHC class II molecules (BD
Bioscience), and measured by flow cytometry using a FACS Canto II analyzer (BD Bioscience) and FlowJo software (FlowJo, USA). Phagocytic activity was measured using a phagocytosis assay kit (IgG-FITC) from Cayman Chemicals (USA). Cells were cultured in RPMI 1640 medium (HyClone, USA) containing 10\% fetal bovine serum (FBS; HyClone), antibiotic-antimycotic agents (Gibco, USA) (complete medium) with or without LPS (Sigma-Aldrich, USA) for $24 \mathrm{hr}$, and secreted cytokines in the supernatant were measured using specific ELISAs.

Culture of splenocytes Splenocytes were prepared as described (Socolovky et al., 2001). Cells were stained with fluorescent-labeled antibodies against CD19, CD69, CD4, CD8, IFN- $\gamma$, IL-4, CD25, and Foxp3 (BD Bioscience), and analyzed by flow cytometry. To measure the proportions of T-cell subsets, splenocytes were cultured in the presence of phorbol 12-myristate 13-acetate, ionomycin, and BD GolgiStop (BD Bioscience) for $4 \mathrm{hr}$. Cells were stained with CD4-FITC at $4^{\circ} \mathrm{C}$ for $30 \mathrm{~min}$, washed with $3 \%$ FBS in PBS, fixed and permeabilized using a Cytofix/Cytoperm TM Fixation/Permeabilization Kit (BD Bioscience), and then intracellularly stained with prelabeled IL- 4 or IFN- $\gamma$ (BioLegend, USA). For T-cell stimulation, cells were incubated with antibodies against CD3 and CD28 or Influenza A virus (A/California/04/2009 (H1N1)) nucleoprotein (NP) peptide (ASNENVETM) for $48 \mathrm{hr}$ (eBioscience, USA), and supernatants were used for quantifying cytokines by ELISA. Cells were stained with CFSE (eBioscience) and cultured in the presence of anti-CD3 and anti-CD28 antibodies for 4 days, and the proportion of mitotic cells was determined by flow cytometry.

Enzyme-linked immunosorbent cytokine assay Mouse IFN- $\gamma$, IL-4, IL-2, IL-6, and TNF- $\alpha$ ELISA kits were purchased from eBioscience, and cytokines in the culture supernatant were measured as described in the manufacturer's protocol. To titrate influenzaspecific antibodies, sera were prepared weekly, and samples were prepared by serial dilution from 1:1,000 to 1:64,000 in PBS containing $1 \%$ bovine serum albumin (PBSA). Enzyme immunoassay 96-well plates (Corning Life Sciences, USA) were coated with $1 \mu \mathrm{g}$ of CBV (SK Chemicals) at $4 \mathrm{~B}^{\circ} \mathrm{C}$ overnight. Nonspecific binding sites were blocked with $1 \%$ PBSA for $1 \mathrm{~h}$ at room temperature (RT), and then 50 ( 1 of an arbitrary standard was added (serum from one of the mice vaccinated against CBV). Samples were loaded into each well and incubated at $4^{\circ} \mathrm{C}$ for $24 \mathrm{hr}$. Plates were washed three times with PBS containing 0.05\% Tween 20 and horseradish peroxidase-conjugated goat anti-mouse IgG was added (Bethyl Laboratories, USA) at RT for $1 \mathrm{hr}$. After washing five times, plates were developed using a chromogenic 3,3',5,5'-tetramethylbenzidine substrate (BD Bioscience), and the reaction was stopped with $2 \mathrm{~N}$ $\mathrm{H}_{2} \mathrm{SO}_{4}$. Optical density was determined at $450 \mathrm{~nm}$ using a Multiskan EX spectrophotometer (Thermo Fisher Scientific, USA).

Statistical analysis Statistical analysis and graphic presentation were conducted using GraphPad Prism 5.01 software (GraphPad, USA). All data are presented as the mean \pm standard error of the mean. Differences between means were determined using Student's $t$ test and one-way analysis of variance (ANOVA); $P<0.05$ was regarded as significant. 


\section{Results}

The activation state of freshly isolated immune cells from obese mice was similar to those from non-obese mice

To generate a model of diet-induced obesity (DIO), we fed mice a $60 \%$ HFD and checked body weight and food intake weekly, with time 0 indicating the start point of feeding. Although overall food intake was lower in mice from the HFD group (data not shown), the mean body weight increased significantly compared with mice from the RFD group. After 10 weeks, epididymal fat mass in mice fed the HFD had increased four-fold compared with the RFD group, and the overall fat mass of mice fed the HFD was increased. The liver weights were similar between mice fed the HFD and RFD, but the HFD induced the accumulation of lipid droplets in hepatocytes (Fig. 1a,b; Supplemental Fig. S1). To compare the state of activation of immune cells between diets, we analyzed activated B cells, T cells, and macrophages from the mice of both groups. The percentage of cells expressing CD69, an early marker of activation in B and T cells, was similar in both groups (Fig. 1c). The population of Th1 cells in mice from the HFD group was higher, but the population of Th2 cells was not, compared with the RFD group. Incidentally, the population of Treg cells in mice from the HFD group was slightly reduced compared with the RFD group (Fig. 1d). As found for CD69 in T and B lymphocytes, the proportion of macrophages expressing CD86 was similar in mice from both groups (Fig. 1e).

T cells isolated from obese animals reacted normally to in vitro stimuli

$\mathrm{T}$ cells are important components of the cellular and humoral immune responses (Janeway et al., 2001). To analyze the activity of $\mathrm{T}$ cells in obese mice, we isolated splenocytes from mice fed either diet and investigated their capacity for proliferation and cytokine production in response to $\mathrm{CD} 3$ and $\mathrm{CD} 28$ stimulation in vitro. Carboxyfluorescein succinimidyl ester (CFSE)-labeled T cells from mice fed either diet were stimulated with CD3 and CD28

(a)

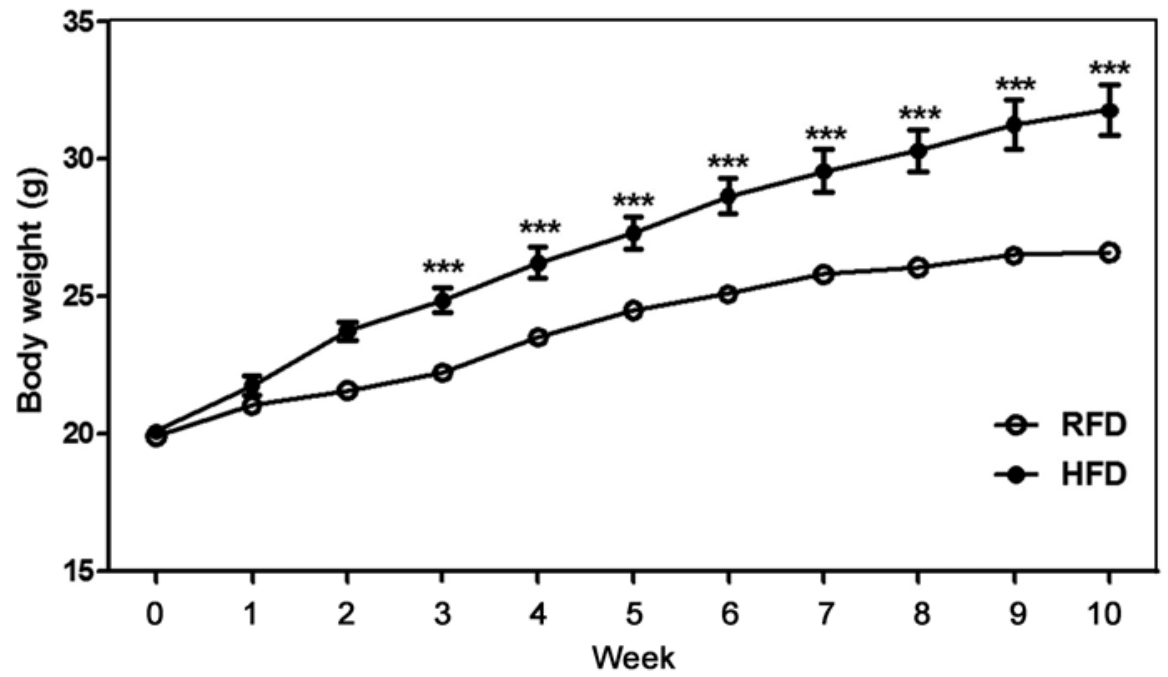

(b)
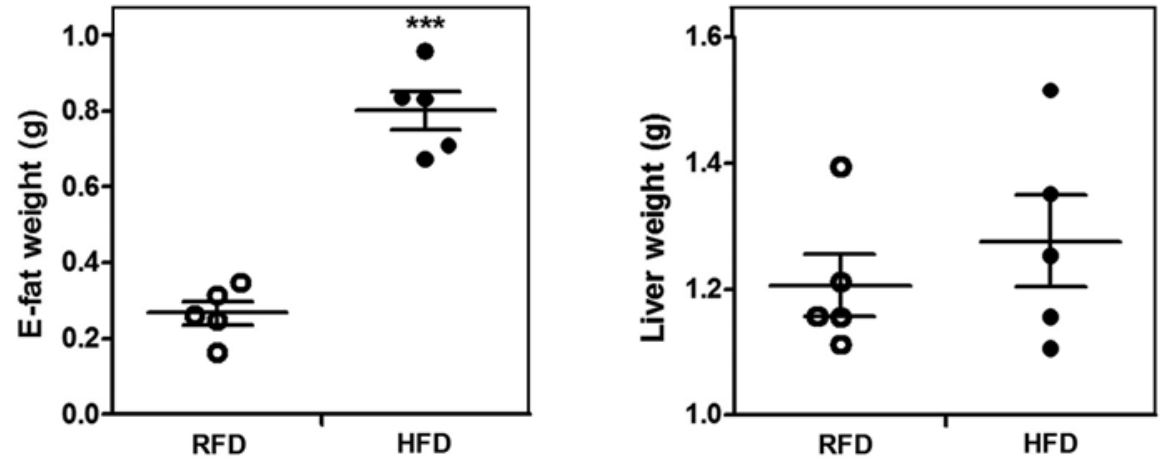

Fig. 1 
(c)
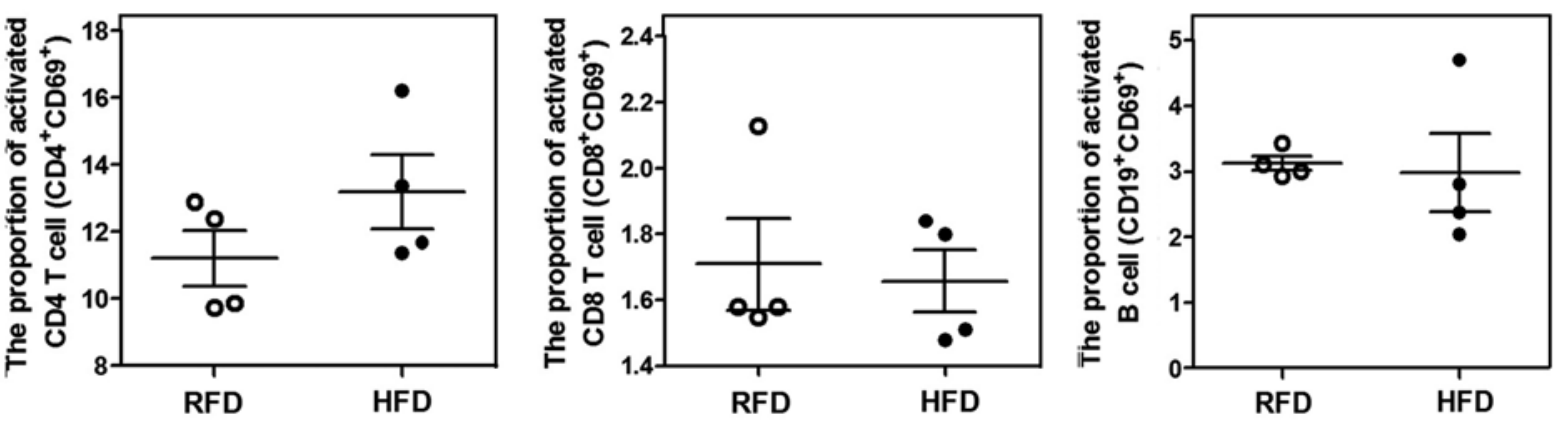

(d)
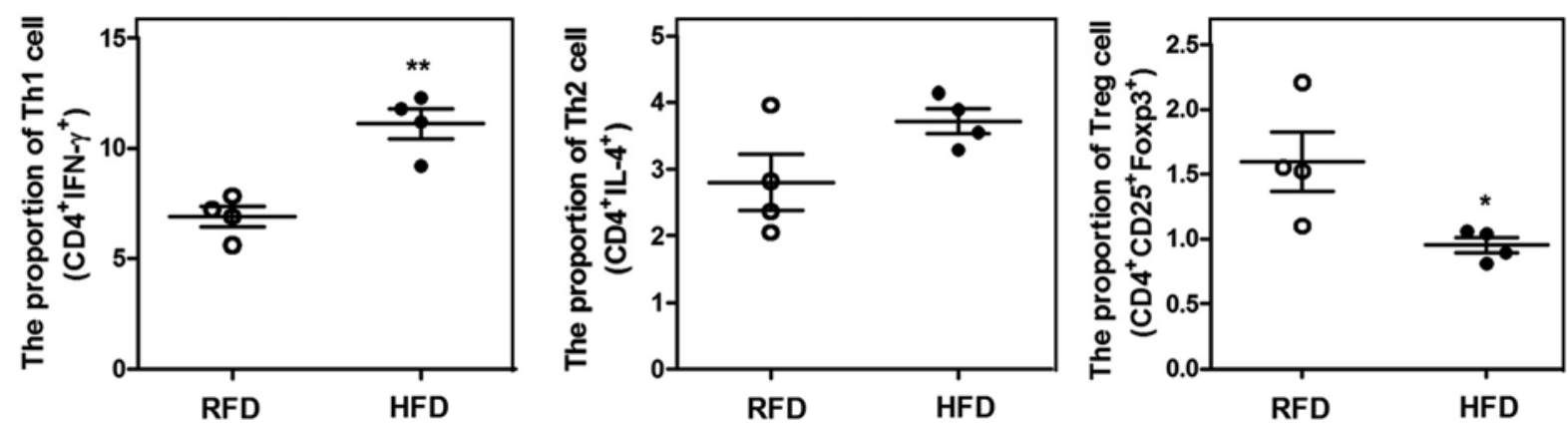

(e)

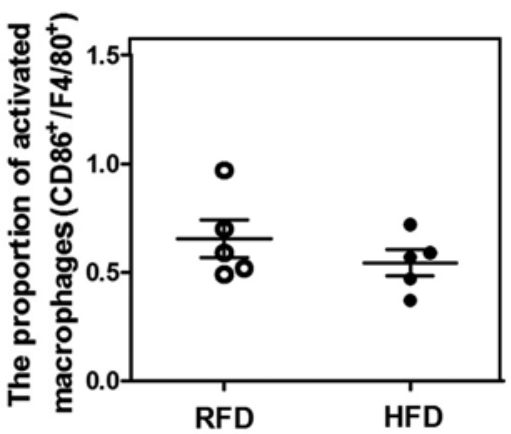

Fig. 1

The HFD increased both body and epididymal fat weights but had no effect on the steady state of immune cells C57BL/6 mice were fed a 60\% (HFD) or 5\% (RFD) fat diet for 10 weeks. (a, b) Changes in body weight (a) and in the weights of epididymal fat and liver at 10 weeks (b) were measured ( $\mathrm{n}=5$ /group). (c) Freshly isolated splenocytes from both groups were stained with fluorescent-labeled antibodies against CD4, CD8, CD19, and CD69, and then analyzed by flow cytometry to determine the proportions of activated populations. (d) Splenocytes were labeled with antibodies against CD4, IL-4, IFN- $\gamma$, CD25, and Foxp3, and each subpopulation was analyzed as described in (c). (e) The proportions of CD86expressing peritoneal macrophages freshly isolated from both groups of mice were measured by flow cytometry. The data were analyzed using Student's $t$ tests; ${ }^{*} P<0.05 ;{ }^{*} P<0.01 ;{ }^{* * *} P<0.001$.

antibodies for 4 days, and the proportion of mitotic cells was compared. Fig. 2 a shows that the proliferative capacity of CD4 T cells was similar for cells from mice in both groups. Moreover, the production of interferon gamma (IFN- $\gamma$ ), IL-2, and IL-4 wasß increased by stimulation but did not differ significantly between splenocytes from mice in either group (Fig. 2b).
Functionality of macrophages was diminished after dietinduced obesity

Macrophages are phagocytes and are at the forefront of the host's defense against infection (Janeway et al., 2001). First, we compared the phagocytic activity of peritoneal macrophages isolated from mice in both groups. Unexpectedly, macrophages 
(a)

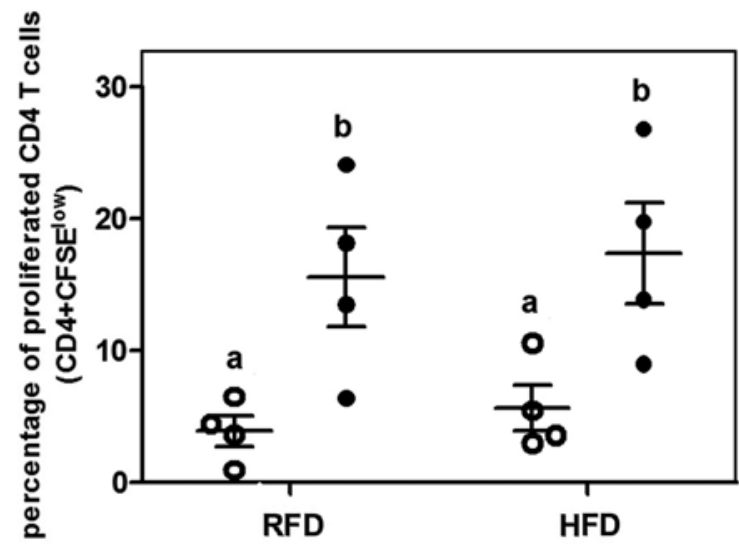

- media

- CD3+CD28

(b)
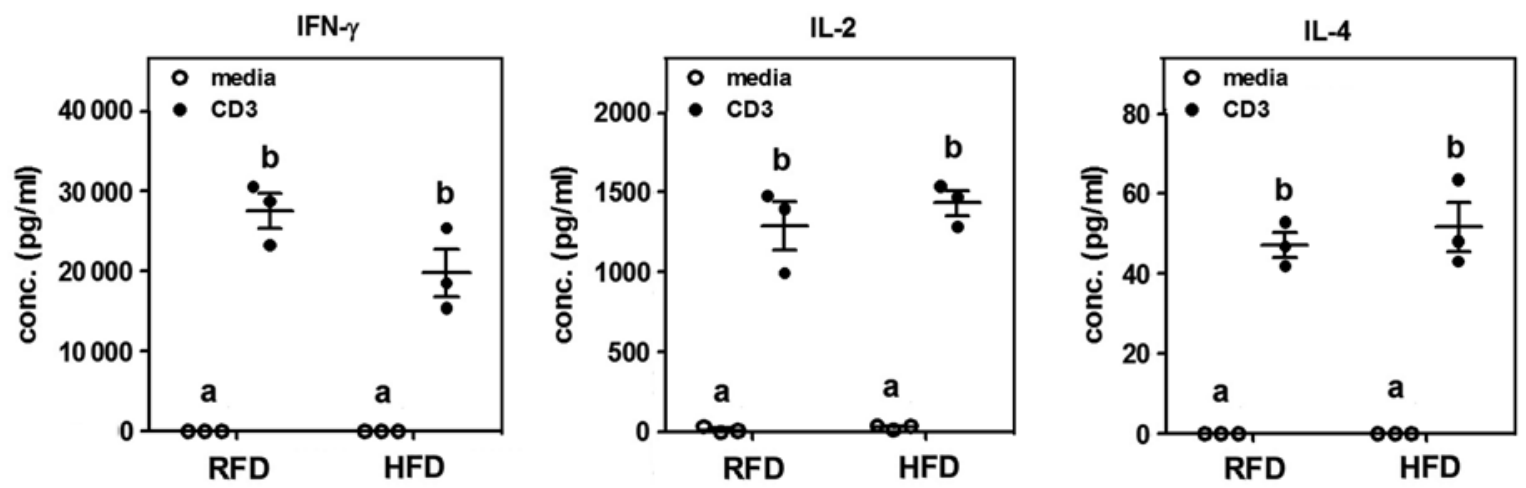

Fig. 2

The HFD had no effect on T-cell responsiveness to CD3 or CD28 stimulation

(a) CFSE-labeled splenocytes from HFD and RFD groups were cultured with anti-CD3 and anti-CD28 antibodies ( $1 \mu \mathrm{g} / \mathrm{ml})$ for $4 \mathrm{days}$, and then the percentages of proliferative $\mathrm{CD} 4^{+}$cells were analyzed using flow cytometry. (b) Splenocytes from the both groups were cultured with anti-CD3 and antiCD28 antibodies $(1 \mu \mathrm{g} / \mathrm{ml})$ for $48 \mathrm{hr}$. The concentrations of IFN- $\gamma$, IL-2, or IL-4 in supernatants were measured using specific ELISAs.

from mice in the HFD group took up more latex beads coated with fluorescein isothiocyanate (FITC)-labeled rabbit IgG than those from the RFD group (Fig. 3a), suggesting that the phagocytic activity of macrophages from mice in the HFD group was higher than those from the RFD group. However, the level of expression of major histocompatibility (MHC) class II antigens on the macrophage surface and the proportion of macrophages expressing MHC class II molecules were similar in both groups (Fig. 3b). Interestingly, freshly isolated macrophages showed very low levels of expression of CD86 (Fig. 1e), but CD86 expression levels were increased when cells were cultured in vitro, and were further increased by lipopolysaccharide (LPS) stimulation (Fig. 3c). However, the basal and the LPS-induced CD86 levels in macrophages from mice in the HFD group were reduced compared with those in macrophages from the RFD group (Fig. 3c). In addition, cytokine production of macrophages induced by LPS also decreased in the HFD group
(Fig. 3d). Although BALB/c mice fed the HFD had a slightly higher body weight than the C57BL/6 mice fed the HFD, it was not dramatically increased compared with C57BL/6 mice (Supplemental Fig. S2 and Fig. 1a). However, the effect of the HFD on the activity of macrophages from $\mathrm{BALB} / \mathrm{c}$ mice was the same as in $\mathrm{C} 57 \mathrm{BL} / 6$ mice (Fig. 3e), meaning that the functionality of macrophages may be abnormal in obese animals regardless of the mouse strain.

Vaccination-induced antibody production was decreased in obese animals

We measured the antibody level in DIO mice vaccinated with an inactivated influenza vaccine, made using a cell culture method (SKYCellflu, SK Chemicals; Seongnam, S. Korea). After 10 weeks on a HFD, we injected cell-culture-based vaccine $(\mathrm{CBV})$ into the inner thighs of the hind legs of mice and 
measured serum levels of vaccine-specific total IgG antibodies by enzyme-linked immunosorbent assay (ELISA) weekly for 8 weeks. Although the concentration of specific antibodies was slightly higher in mice from the HFD group at 1 week, this reversed gradually and by 3 weeks was lower than that in the RFD group, whereas the serum antibody level in mice from the RFD group continued to increase for 5 weeks after vaccination (Fig. 4a). We could not detect a vaccine-specific $\mathrm{T}$ cell response in the spleen (data not shown). However, responsiveness to polyclonal activation of splenic $\mathrm{T}$ cells remained intact in the HFD group (Fig. 4b).

\section{Discussion}

Obesity is a metabolic disease that is continuing to increase worldwide. Since the pandemic of H1N1 influenza virus in 2009, the relationship between obesity and the pathogenicity of viral infection has been investigated intensively, showing that the protective immune response is diminished in obesity (Kim et al., 2009; Karlsson et al., 2010a,b; Sheridan et al., 2012; Park et al., 2014). A previous study showed that mice fed an HFD showed lower levels of neutralizing antibody titers against influenza vaccine than did RFD mice because of obesity-induced chronic inflammation (Park et al., 2014). Moreover, humans with a high body mass index showed quick declines in anti-influenza antibody titers (Sheridan et al., 2012). These low titers might be caused by defective T-cell function (Karlsson et al., 2010a,b). In the present study, we examined the activity of immune cells isolated from mice induced to be obese with an HFD to clarify the immunological environment of obesity.

Neutralizing antibodies are the best effectors of host defense against viral infections. They prevent infection of cells by blocking the binding of viruses to cell surface receptors (Janeway et al., 2001). Vaccine-induced antibody production is lower in obese mice than it is in normal mice (Kim et al., 2009; Park et al., 2014). Moreover, Sheridan et al. (2012) also showed that antibody production was intact but maintenance was deficient in obese individuals. Here we found that the initial serum antibody concentration was similar in mice fed either diet, but the rate of increase was lower in mice from the HFD group and the rate of degradation was similar in both groups. These findings indicate that insufficient production of antibody significantly influenced the serum level of antibodies between mice fed either diet. Although B cells are the main producers of antibodies, many other immune cells are involved in antibody

(a)

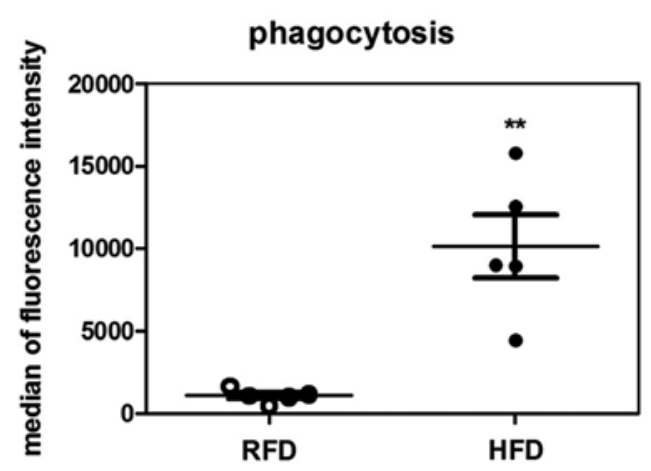

(b)
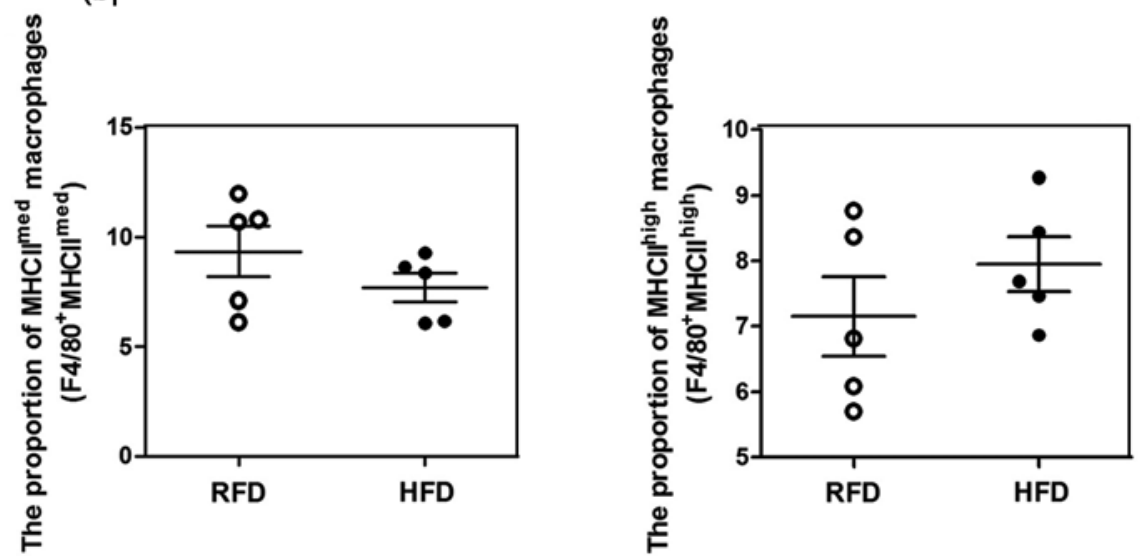

Fig. 3 
(c)

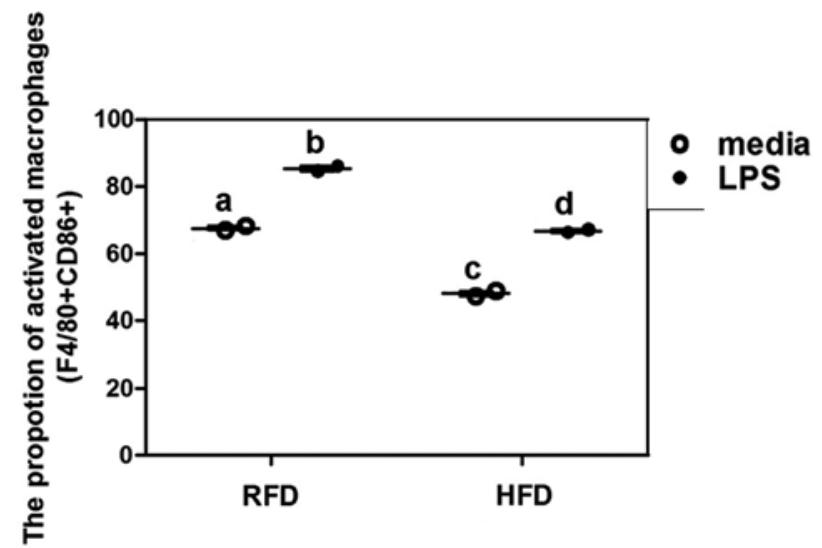

(d)

IL-6

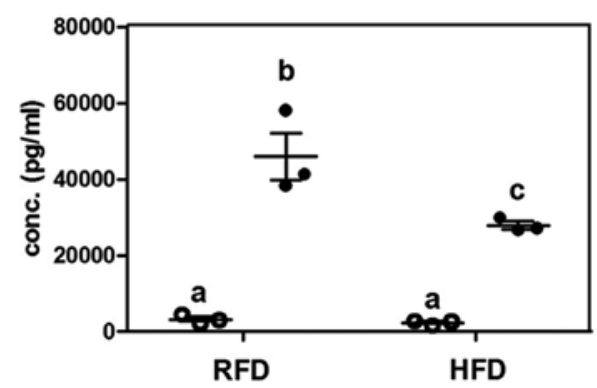

(e)

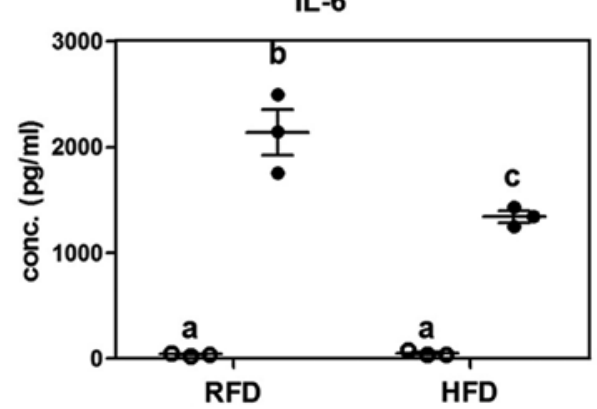

TNF- $\alpha$

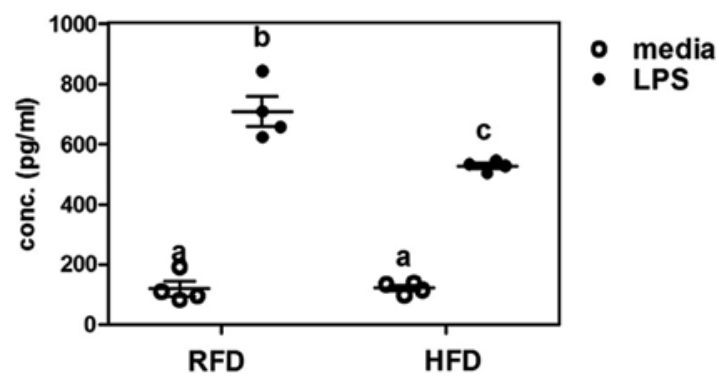

TNF- $\alpha$

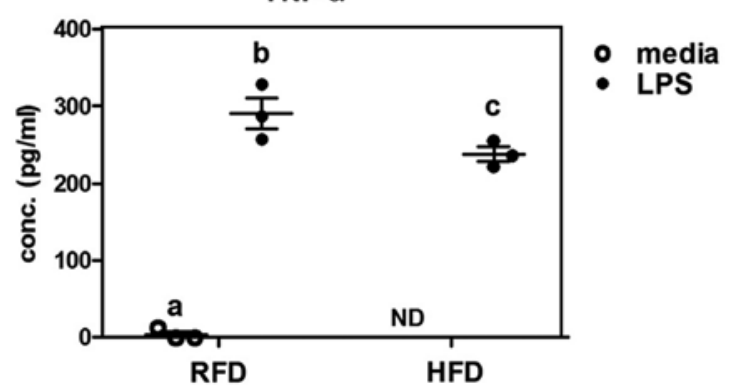

Fig. 3

Activation of macrophages by LPS and cytokine secretions were decreased in the HFD group

(a) Peritoneal macrophages from HFD and RFD groups were cultured in the presence of FITC-conjugated rabbit IgG for 24 hr, and phagocytic activities (FITC levels) were measured by flow cytometry. (b) Macrophages were stained with an anti-MHC class II (I-A/I-E) antibody, and the proportions of macrophages expressing MHC class II molecules were compared. (c, d) Macrophages were cultured with LPS (100 ng/ml) for 24 hr. The percentages of activated macrophages $\left(\mathrm{CD}^{+} 6^{+}, \mathrm{c}\right)$, pooled from five mice, were measured using flow cytometry, and the concentrations of IL- 6 and TNF- $\alpha$ (d) in the supernatant were measured using specific ELISAs. (e) BALB/c mice were fed a $60 \%$ (HFD) or 5\% (RFD) fat diet for 10 weeks. Cytokine production levels by LPS-stimulated macrophages were measured as in (d). The data were analyzed using Student $t$ test; ${ }^{* *} P<0.01$ and a one-way ANOVA followed by a Tukey post-hoc test; different letters indicate statistically significant differences between the groups.

production. Macrophages and dendritic cells take up antigen and produce peptides via antigen processing, and then present these peptides to $\mathrm{T}$ cells through an MHC-peptide complex. Activated T cells differentiate to form effector or memory cells and help other cells by secreting cytokines and costimulatory molecules, such as CD40. When a T cell encounters a cognate $\mathrm{B}$ cell, they help in the proliferation and differentiation of that B cell, which ultimately becomes a differentiated plasma cell producing antibodies (Janeway et al., 2001). We found that the activation state of immune cells in obese mice did not differ 
(a)

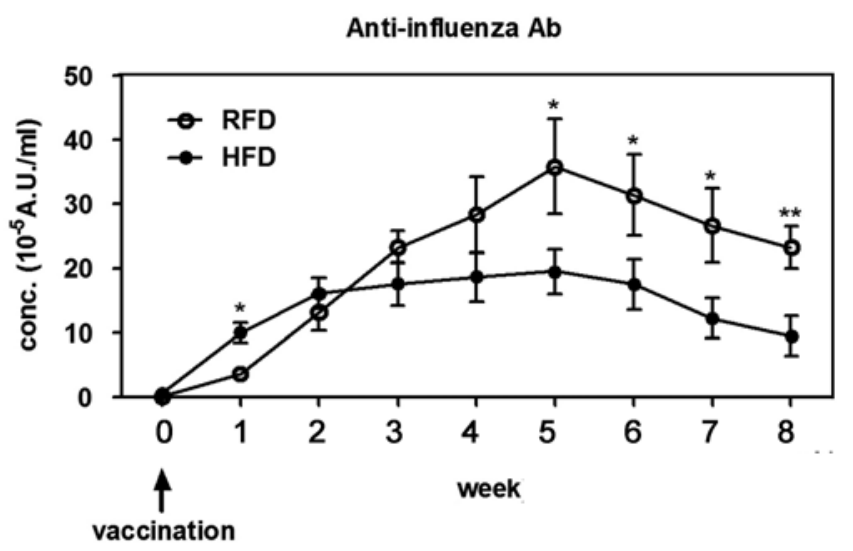

(b)
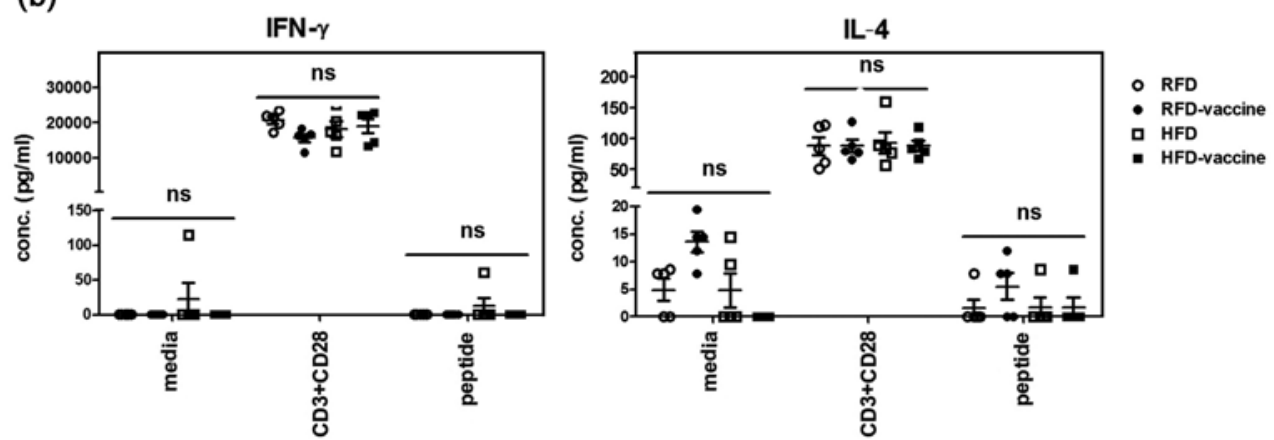

Fig. 4

Vaccine-induced antibody production was reduced in DIO mice

C57BL/6 mice were fed an HFD or an RFD for 10 weeks. (a) Antigen-specific total IgG was measured in sera from the both groups vaccinated with CBV at 10 weeks. (b) Reactivity to the anti-CD3 and anti-CD28 antibodies $(1 \mu \mathrm{g} / \mathrm{ml})$, or influenza NP peptide ( $1 \mu \mathrm{g})$ of T cells was analyzed by of IFN- $\gamma$, IL- 4 ELISAs. The data were analyzed using Student $t$ test; ${ }^{\star} P<0.05 ;{ }^{\star \star} P<0.01 ;{ }^{\star \star \star} P<0.001$.

significantly from those of lean mice and that the reactivity of T cells was similar in both groups. Even though we did not observe an antigen-specific response, we conclude that $\mathrm{T}$ cells in DIO mice have normal activity. The influenza-induced memory $\mathrm{T}$ cell response is reduced in DIO animals (Karlsson et al., 2010a,b). However, this might be the result of other influences - such as reduced antigen presentation or specificity of the antigen - rather than T-cell intrinsic activity.

Unlike $\mathrm{T}$ cells, macrophages from mice fed the HFD or RFD exhibited many functional differences. Macrophages are dedicated phagocytes and antigen-presenting cells, and play roles in the innate and adaptive immune systems. Following infection, macrophages not only take up and present antigens but also secrete proinflammatory cytokines including TNF- $\alpha$ and IL- 1 to activate immune cells and kill pathogens (Murray and Wynn, 2011). Here, the population of activated macrophages in vivo seems to be unchanged between mice in the HFD and RFD groups. However, phagocytic activity in vitro was higher in macrophages from mice in the HFD group, whereas MHC levels were similar in both groups. These in vivo data do not seem to be matched by the in vitro data. It is a limitation of the study that macrophage phagocytic activity was not directly measured in vivo. Moreover, the population of activated macrophages does not directly represent phagocytic activity. Thus, this finding suggests that antigen presentation might be unchanged by obesity. However, when macrophages were stimulated with LPS, cytokine production was lower in mice in the HFD group compared with those from the RFD group. This indicates that although macrophage activity in vivo was seemingly unchanged, obesity-induced chronic inflammation might induce macrophage tolerance, leading to inactivation of macrophages despite the presence of an external stimulus such as LPS or vaccine. Moreover, the HFD group showed increased proportions of Th1 cells and reduced proportions of Treg cells, which might contribute to the induction of inflammation. Taken together, obesity could induce an abnormality in macrophages that can influence $T$ cell behavior, thereby affecting antibody production. We could not obtain direct evidence of any reduced ability of macrophages from the HFD group in activated T cells. Further study is needed 
to determine the exact mechanism of the inhibitory effect of obesity on the vaccine-induced humoral immune response.

In summary, we found that obesity induced by an HFD did not affect the phenotype of immune cells in a steady state, but it reduced CD86 expression and cytokine production of activated macrophages, which might contribute to a decreased immune response against vaccine. Therefore, recovery of macrophage functionality might help in increasing the efficacy of vaccination in obese individuals.

Supplementary information is available in the online version of the paper.

Acknowledgements. This work was supported by the Catholic University of Korea, Research Fund, 2016, the Korean Healthcare Technology R\&D project of the Ministry of Health \& Welfare (HI13C0826), a grant from the KHIDI, funded by the Ministry of Health \& Welfare, Republic of Korea (grant number: HI15C2955), and Basic Science Research Program through the NRF funded by the Ministry of Science, ICT \& Future Planning (NRF-2015M3A9B5030157).

\section{References}

Cho W, Nam JH (2014): Is Obesity one of physiological factors which exert influenza virus-induced pathology and vaccine efficacy? J. Bacteriol. Virol. 44, 226-235. http://dx.doi. org/10.4167/jbv.2014.44.3.226

Hotamisligil GS, Arner P, Caro JF, Atkinson RL, Spiegelman BM (1995): Increased adipose tissue expression of tumor necrosis factor-alpha in human obesity and insulin resistance. J. Clin. Invest. 95, 2409-2415. http://dx.doi. org/10.1172/JCI117936

Ikejima S, Sasaki S, Sashinami H, Mori F, Ogawa Y, Nakamura T, Abe Y, Wakabayashi K, Suda T, Nakane A (2005): Impairment of host resistance to Listeria monocytogenes infection in liver of $\mathrm{db} / \mathrm{db}$ and ob/ob mice. Diabetes 54, 182-189. http://dx.doi.org/10.2337/diabetes.54.1.182

Janeway CA Jr, Travers P, Walport M, Shlomchik MJ (2001): Immunobiology. 5th ed. Garland Science, New York.

Jo J, Gavrilova O, Pack S, Jou W, Mullen S, Sumner AE, Cushman SW, Periwal V (2009): Hypertrophy and/or hyperplasia: dynamics of adipose tissue growth. PLoS Comput. Biol. 5, e1000324. http://dx.doi.org/10.1371/journal.pcbi.1000324

Kanneganti TD, Dixit VD. (2012): Immunological complications of obesity. Nat. Immunol. 13, 707-712. http://dx.doi. org/10.1038/ni.2343

Karlsson EA, Sheridan PA, Beck MA (2010): Diet-induced obesity impairs the $\mathrm{T}$ cell memory response to influenza virus infection. J. Immunol. 184, 3127-3133. http://dx.doi. org/10.4049/jimmunol.0903220

Karlsson EA, Sheridan PA, Beck MA (2010): Diet-induced obesity in mice reduces the maintenance of influenza-specific CD8+ memory T cells. J. Nutr. 140, 1691-1697. http:// dx.doi.org/10.3945/jn. 110.123653
Kim YH, Kim JK, Kim DJ, Nam JH, Shim SM, Choi YK, Lee CH, Poo $\mathrm{H}$ (2009): Diet-induced obesity dramatically reduces the efficacy of a pandemic $\mathrm{H} 1 \mathrm{~N} 1$ vaccine in a mouse model. J. Infect. Dis. 205, 244-251. http://dx.doi.org/10.1093/infdis/jir731

Loffreda S, Yang SQ, Lin HZ, Karp CL, Brengman ML, Wang DJ, Klein AS, Bulkley GB, Bao C, Noble PW, Lane MD, Diehl AM (1998): Leptin regulates proinflammatory immune responses. FASEB J. 12, 57-65.

Lord GM, Matarese G, Howard JK, Baker RJ, Bloom SR, Lechler RI (1998): Leptin modulates the T-cell immune response and reverses starvation-induced immunosuppression. Nature 394, 897-901. http://dx.doi.org/10.1038/29795

Macia L, Delacre M, Abboud G, Ouk TS, Delanoye A, Verwaerde C, Saule P, Wolowczuk I (2006): Impairment of dendritic cell functionality and steady-state number in obese mice. J. Immunol. 177, 5997-6006. http://dx.doi.org/10.4049/ jimmunol.177.9.5997

Mattioli B, Straface E, Quaranta MG, Giordani L, Viora M (2005): Leptin promotes differentiation and survival of human dendritic cells and licenses them for Th1 priming. J. Immunol. 174, 6820-6828. http://dx.doi.org/10.4049/ jimmunol.174.11.6820

Murray PJ, Wynn TA (2011): Protective and pathogenic functions of macrophage subsets. Nat. Rev. Immunol. 11, 723-737. http://dx.doi.org/10.1038/nri3073

Park HL, Shim SH, Lee EY, Cho W, Park S, Jeon HJ, Ahn SY, Kim H, $\mathrm{Nam} \mathrm{JH} \mathrm{(2014):} \mathrm{Obesity-induced} \mathrm{chronic} \mathrm{inflammation} \mathrm{is}$ associated with the reduced efficacy of influenza vaccine. Hum. Vaccin. Immunother. 10, 1181-1186. http://dx.doi. org $/ 10.4161 / h v .28332$

Plotkin BJ, Paulson D (1996): Zucker rat (fa/fa), a model for the study of immune function in type-II diabetes mellitus: effect of exercise and caloric restriction on the phagocytic activity of macrophages. Lab. Anim. Sci. 46, 682-684.

Rosen ED, Spiegelman BM (2006): Adipocytes as regulators of energy balance and glucose homeostasis. Nature 444, 847-853. http://dx.doi.org/10.1038/nature05483

Sheridan PA, Paich HA, Handy J, Karlsson EA, Hudgens MG, Sammon AB, Holland LA, Weir S, Noah TL, Beck MA (2012): Obesity is associated with impaired immune response to influenza vaccination in humans. Int. J. Obes. 36 , 1072-1077. http://dx.doi.org/10.1038/ijo.2011.208

Smith AG, Sheridan PA, Harp JB, Beck MA (2007): Diet-induced obese mice have increased mortality and altered immune responses when infected with influenza virus. J. Nutr. 137, 1236-1243.

Socolovsky M, Nam H, Fleming MD, Haase VH, Brugnara C, Lodish HF (2001): Ineffective erythropoiesis in Stat 5a-/-5b-/-mice due to decreased survival of early erythroblasts. Blood 98, 3261-3273. http://dx.doi.org/10.1182/blood.V98.12.3261

Webb SR, Loria RM, Madge GE, Kibrick S (1976): Susceptibility of mice to group B coxsackie virus is influenced by the diabetic gene. J. Exp. Med. 143, 1239-1248. http://dx.doi. org/10.1084/jem.143.5.1239

Weisberg SP, McCann D, Desai M, Rosenbaum M, Leibel RL, Ferrante AW, Jr (2003): Obesity is associated with macrophage accumulation in adipose tissue. J. Clin. Invest. 112, 1796-1808. http://dx.doi.org/10.1172/JCI200319246 